\title{
FREGE CONTRA LA CONCEPCIÓN BOOLEANA DE LA LOGICA
}

Pilar CASTRILLO

UNED.Dpto. de Lógica y Filosofia de la Ciencia

La publicación de la Begriffsscrift de Frege constituye, como es sabido, uno de los hitos más importantes de toda la historia de la lógica. De hecho señala el inicio de una nueva época en el desarrollo de esta disciplina. Sin embargo, la novedad de las aportaciones en ella contenidas y el proyecto al que obedecía escaparon por completo a la comprensiôn de sus contemporáneos, tanto matemáticos como filósofos. Como él mismo señalaría más tarde, nada cabia esperar de los primeros que, al tropezar con expresiones lógicas como "concepto", "juicio", etc., se excusaban diciendo "Methaphysica sunt, non leguntur", pero tampoco mucho más de los segundos, los cuales, ante la presencia de fórmulas, enseguida se disculpaban con un "Mathematica sunt, non leguntur". Es evidente que la dificultad técnica que encerraba el material expuesto en esta obra constituia una traba para la comprensión de la misma, pero ésta no es sino una sola de las razones de la mala acogida que se le dispensó, cuya causa habría que buscarla también en la falta de explicaciones por parte de su autor acerca de cosas tales como lo que se proponia hacer en la obra, la vinculación que este proyecto tenia con el leibniziano o los presupuestos filosóficos sobre los que se asentaba. El hecho de que dos de los representantes más destacados de la lógica en aquel momento, $\mathrm{J}$. Venn en Gran Bretaña y E. Schröder en Alemania criticaran la obra, afirmando no haber hallado en ella más que un modo mucho más engorroso y complicado de lograr lo que, después de todo, Boole habia conseguido hacer de forma mucho más sencilla en The Laws of Thought, indujo a nuestro autor a dedicar algunos esfuerzos adicionales a explicar por fin lo que se habia propuesto

Endaxa: Series Filosóficas $n^{9} 1,1993$, INED, Madrid:

Pilar CASTRILLO. Frege contra la concepción boolecula de la lógica:

pp.217-238. 
hacer alli y en especial a dar cuenta de las diferencias que separaban a su proyecto del de Boole y los booleanos.

Fruto de estos esfuerzos son una serie de trabajos compuestos durante los tres años que siguieron a la aparición de Begriffsscrift. alguno de los cuales ha permanecido inédito hasta la publicación, en 1969. de los Nachgelassene Schiften de Frege. Este es el caso de "Booles rechnende Logik und die Begriffsscrift"1, que es el más largo e interesante de todos ellos, pues es en el que explica las motivaciones de su nueva lógica con mayor detalle, pero el resto de los que urdió en esta época responden al mismo objetivo de esclarecer las propiedades y propósitos de su propia notación en comparación con los de la de Boole ${ }^{2}$. El análisis de estos trabajos, a los que nunca se ha prestado demasiada atención, encierra, en mi opinión, un gran interés no solo para esclarecer el pensamiento lógico y filosófico de Frege en este primer estadio de su desarrollo, sino también para llegar a una más clara comprensión de este decisivo periodo de la lógica moderna.

\section{Lógica como cálculo y logica como lengua característica.}

Desde el comienzo de su carrera, a Frege le había venido preocupando una cuestión: la de la fundamentación de la matemática. Lo que él se habia propuesto no era en realidad sino presentar las pruebas de la aritmética de una forma tan rigurosa y precisa que en las cadenas de razonamiento "no pudiera colarse inadvertidamente nada intuitivo" ${ }^{3}$. Para lograr este objetivo, Frege pensó en

\footnotetext{
${ }^{1}$ Nachgelassene Schriften, ed. H. Hennes et al., Hamburg, Meiner, 1969.pp. 9-53. Hay también versión inglesa. Posthumous Writings, ed. H. Hermes et al., Chicago. University of Chicago Press, 1979. A partir de ahora me referiré a esta obra como NS.

${ }^{2}$ Entre ellos cabe destacar "Über die wissenschaftliche Berechtigung einer Begriffsschrift". ZPPK 81 (1882). pp. 48-56 y "Über den Zweck der Begriffsscrift". Sitzungsberichte der Jenaischen Gesellchaft für Medicin und Naturwissenschaft. JZN 16 (1882-83, pp. 1-10. Las versiones inglesas de ambos. "On the Scientific Justification of a Conceptual Notation" $y$ "On the aim of the 'Conceptual Notation"', se hallan recogidas en Conceptual Notation and related articles. ed. T. W. Bynum. pp. 83-90 y 90-101, respectivamente. A partir de ahora me referiré a esta obra como $C N$.

${ }^{3}$ Begriffsscrift. Prefacio. Kleine Schriften. ed. 1. Angelelli. Hildesheim. 1967.
} 
un principio en servirse del lenguaje ordinario (en concreto, del alemán), pero se encontró con que la ambigüedad e inexactitud del mismo lo hacian inservible para presentar las pruebas con los requisitos de exactitud y rigor que se había fijado. Esto le llevó a poner sus ojos en el ideal leibniziano de lenguaje universal, ideal que conocia por el ensayo de Trendelenburg "Über Leibnizens Entwurf einer allgemeinen Characteristik"4, a través del cual dicho autor había popularizado este y otros aspectos de la obra leibniziana poco conocidos entonces. Fue asi como se vio llevado a desarrollar un lenguaje simbólico al que bautizó con el nombre de "Begriffsscrift", notación conceptual, toda vez que lo que con él pretendia era poder expresar los contenidos conceptuales (Begriffliche Inhaltel de los juicios de la matemática y de la lógica. Esta denominación, aunque en teoria plenamente justificada, en la práctica resultó, sin embargo, mas bien desafortunada, ya que, al no ser el simbolo del concepto sino un término, propiciaba una interpretación de su sistema como lo que en realidad no era: como una pura lógica de términos, i.e., como un sistema de la indole de los construidos por Boole y sus seguidores. Asi es como de hecho lo juzgará Schröder en la dura critica a que somete a la obra al año siguiente de su publicación ${ }^{5}$, critica en la que, tras de lamentarse de que el título, "Begriffsscrift", promete demasiado o, mejor aún, "no se corresponde en absoluto con el contenido" del libro, señala

En lugar de propender a una caracteristica universal, el presente trabajo (tal vez sin que el propio autor sea consciente de ello) propende al "calculus ratiocinator" de Leibniz. En esta dirección este libro hace un avance que habria que considerar muy estimable, si una buena parte de lo que intenta no hubiera

\footnotetext{
${ }^{4}$ Historische Beiträge zur Philosophie. vol. 3, Berlin. 1867. pp. 1-47.

5 "Review of Frege's Conceptual Notation", en CN, pp. 218-232. La critica se publicó originariamente en Zeitschrift für Mathematik und Physik, 25 (1880), pp. 81 94. Hans D. Sluga, en "Frege against the booleans". Notre Dame Journal of Logic 28 (1987). pp. 80-98, señala que la crítica de Schröder. entonces en posesión ya de una posición académica estable. fue innecesariamente hostll y pudo haber dañado la carrera académica de Frege. En cambio. Frege, que en ocasiones llega incluso a ser sarcástico con sus adversarios. es siempre precavido y moderado cuando arremete contra los booleanos.
} 
sido ya realizado por algún otro y (como mostraré) de una forma sin duda más adecuada ${ }^{6}$.

"Calculus ratiocinator", "lingua characteristica" son los términos de los que también se servirá Frege para expresar las diferencias que, a su juicio, separan su proyecto del de los booleanos, sólo que él va a invertir el orden de aplicación de los mismos, ya que su idea es que las cosas son justamente al revés de como las ha descrito Schröder, i.e..que es él el que se ha acercado al ideal leibniziano de lengua characterica (como él dice), en tanto que Boole no ha logrado más que un calculus. Así, comparando el sistema urdido por Peano con el de los booleanos y con el suyo propio, en un ensayo publicado muy posteriormente, afirma ${ }^{7}$

[El sistema conceptual de Peano] se presenta como un descendiente del cálculo lógico de Boole, pero hay que decir que como distinto de los demás. No digo ésto en un sentido peyorativo; por el contrario considero las divergencias de Boole como mejoras importantes. La lógica de Boole es lógica y nada más. Se ocupa únicamente de la forma lógica. y no de la introducción de un contenido en dicha forma -en tanto que ésta es precisamente la intención del Sr. Peano. En este sentido, su empresa se asemeja más a mi notación conceptual que la lógica de Boole. Desde otro punto de vista, sin embargo, cabe reconocer una más estrecha afinidad entre la lógica booleana y mi notación conceptual, en la medida en que las dos insisten principalmente en la inferencia, en la que no se hace tanto hincapié en el cálculo lógico de Peano. En terminologia leibniziana podriamos decir:la lógica de Boole es un calculus ratiocinator. pero no una lingua characterica ; la lógica matemática de Peano es fundamentalmente una lingua

\footnotetext{
${ }^{6}$ CN. pp. 219-220.

7 "Über die Begriffsschrift das Herrn Peano und meine eigene". publicado inicialmente en 1896, y recogido, en versión inglesa, en Collected Papers on Mathematics, Logic und Philosophy. ed. B. McGuinness. Oxford. Blackwell. p.242.A partir de ahora me referiré a esta obra como $C P$.
} 
characterica y también un calculus ratiocinator, mientras que mi notación conceptual es ambas cosas por igual.

Frege y Schröder tomaron, pues, de la misma fuente los términos en los que expresaron sus diferencias, pero ésto no es todo lo que hay de común entre ellos. sino que, al insistir en que el simbolismo propio constituia una auténtica caracteristica en tanto que el del contrario se quedaba en un mero cálculo. estaban mostrando estar también de acuerdo aún en otra cosa: a saber, en que el simbolismo lógico debe ser un lenguaje caracteristico. Su desacuerdo se iniciaba, pues, con la cuestión de cómo había de ser semejante lenguaje y qué propiedades habia de tener.

Ambos sabian por el ensayo de Trendelenburg que, para Leibniz, el lenguaje caracteristico era un lenguaje en el que la estructura lógica de las aserciones ha de reflejar la estructura de los objetos representados. en tanto que un cálculo no era sino un mecanismo para determinar la verdad de las aserciones, pero ¿era ésto lo que ellos querian decir con semejantes términos. especialmente con el primero? ¿Son Frege y Schröder fieles a las acepciones con que Leibniz los emplea?. En el caso de Schroder, el uso que hace de la expresión 'lingua characteristica' si parece, a primera vista, muy similar al de Leibniz, ya que para él, como para éste. en un lenguaje universal uno ha de ser capaz de "construir todos los conceptos complejos por medio de algunas operaciones simples, completamente determinadas y claramente clasificadas, a partir del menor número posible de conceptos con extensiones claramente delimitadas" ${ }^{\text {" }}$, siendo la principal razón para negarle el calificativo de "lenguaje caracteristico" al sistema fregeano el que no se ajusta a semejante patrón. En cuanto a Frege, no es éste desde luego el sentido en el que él emplea la expresión, y , por tanto, desde este punto de vista, él es el que parece haberse apartado más de Leibniz. Mas hay otro aspecto en los planes de Leibniz para la construcción de un lenguaje universal, al que no en vano, además de characteristica universalis, denominó lingua philosophica, que a Schröder parece habérsele escapado y en el cual, en cambio, repara Frege: el lenguaje lógico, tal como Leibniz lo entiende, requiere no

${ }^{8}$ CN p. 219 
solo razonamiento calculístico, sino también análisis conceptual. El cree, en efecto, que si su sistema se aproxima más que el de los booleanos al ideal leibniziano de lengua universal es porque éstos se han concentrado en un único aspecto: los procedimientos mecánicos por los que se pueden manipular ciertos elementos, cosa que "representa solo una parte de nuestro pensamiento; el todo no puede ser realizado por una máquina o reemplazado por una actividad puramente mecánica"9, en tanto que él, en cambio, no ha descuidado el que la construcción de un lenguaje caracteristico requiere también análisis conceptual. Pero, ¿ Qué particularidades de su proyecto son las que ,en concreto, lo hacen ,a los ojos de Frege, acreedor del nombre de lengua caracteristica y por qué el de Boole solo alcanza la categoría de cálculo?

Hace ya algunos años, el insigne historiador de la lógica, van Heijenoort, en un ensayo que lleva por título "Logic as calcul, logic as language" 10 , señalaba la necesidad de distinguir, para poder comprender el posterior desarrollo de la lógica, y más en concreto la evolución de ésta en la década de los 20 , entre dos corrientes de investigación, la algebraica y la inaugurada por Frege y luego continuada por Russell y Wittgenstein. Van Heijenoort hacia también hincapié en las ventajas heuristicas que a su juicio se seguirian del esclarecimiento de lo que Frege habia querido significar al reservar el membrete de lenguaje caracteristico para su propio sistema de notación y designar el de los booleanos con el nombre de cálculo y , por su parte, aventuró una interpretación que, en lineas generales, es la siguiente: el sistema fregeano es un lenguaje porque para Frege y los logicistas el lenguaje de la lógica es un lenguaje universal, un medio, por tanto, del que no nos podemos salir para hablar de él, lo cual quiere decir que, en esta perspectiva, están ausentes por completo todo tipo de consideraciones metasistemáticas. Para los booleanos el simbolismo lógico es, en cambio, un cálculo, porque ellos lo conciben no como una lengua universal, sino como un sistema susceptible de diversas interpretaciones según los diferentes universos del discurso, cosa que propiciaria el planteamiento de cuestiones de validez de

9 "Booles rechnende..."NS, p. 39.

${ }^{10}$ Synthese 17 (1967), pp. 324-333. 
fórmulas en diferentes dominios. Van Heijenoort sintetizó también lo que, a su juicio, distingue a esta última corriente de la logicista en los cuatro rasgos siguientes:1) una mayor valoración (que en la corriente fregeana) de la lógica de primer orden, en comparación con la de segundo orden; 2) la práctica, heredada de Boole, de tomar en consideración distintos dominios de interpretación; 3) la ausencia de una noción rigurosa de prueba, y 4) la obtención de resultados mediante razonamientos de tipo semántico, aunque no haya todavia un concepto preciso de validez o satisfacción ${ }^{\prime \prime}$. Estos rasgos permitirian , en su opinión, explicar el que un resultado como el de Löwenheim de 1915, con el que se considera que nace la teoria de modelos, impensable dentro de la tradición fregeana. hubiera tenido lugar, en cambio, en la algebraica.

Hoy dia esta tesis de van Heljenoort goza de un reconocimiento prácticamente unánime y se considera que encierra una descripción , en líneas generales, correcta de las condiciones en las que surgió la metamatemática en las primeras décadas de este siglo $^{12}$. Es decir, hay un consenso general en que, siempre que no se carguen demasiado las tintas, es conveniente distinguir entre dos perspectivas que habrian configurado el desarrollo de la lógica hasta su fusión en la década de los 20: una sintáctica, caracteristica de la tradición que se inaugura con Frege y se continúa con Russell y el primer Wittgenstein y otra semántica, peculiar de la línea de investigación inaugurada por Boole y proseguida por Schröder y el primer Peirce. Es dentro del marco de esta última donde, después

\footnotetext{
11 "Set-theretic semantics". Logic Colloquium 76, ed. R.Gandy y M. Hylan. NorthHolland. Amsterdaun. 1977. p 183

${ }^{12}$ Entre los trabajos que han desarrollado esta tesis de van Heijenoort cabe destacar W. D. Goldfarb. "Logic in the twenthies: the nature of quantifier". The Journal of Symbolic Logic 44 (1979, pp.35 1-368:G.H. Moore. "The emergence of first order logic", en Histoty and philosophy of modern mathematics. ed. W. Aspray and P. Kitcher. Minnesota Studies in the Philosophy of Science. Univ. of Minnesota Press. vol XI. 1988. pp. 95-135, y J. Hintikka. "On the development of the modeltheoretical tradition in logical theory". Synthese 77 (1988). pp. 11-26.El más destacado de los disidentes de esta interpretación es $M$. Dummett, quien en su Frege. Philosophy of language (Duckworth. London. Harper and Row, 1973) ha señalado que. en Frege, la interpretación de una fómula del lenguaje de la lógica predicat iva "se obtiene asignando entidades de indole adecuada a las constantes nológicas primitivas que aparecen en la fórmula...|Estel procedimjento es exactamente idéntico al tratamiento semántico de la lógica de predicados" (pp. 89-90).
} 
que se llega a una percepción clara de la lógica de primer orden como un sistema independiente, surge el planteamiento de cuestiones metateóricas. Este tipo de cuestiones resultan en cambio ilegitimas en la perspectiva logicista, pues, como el sistema se entiende como un lenguaje universal, no puede haber punto de vista externo alguno desde el que contemplar y discutir dicho sistema. Mas una cosa es reconocer la validez y pertinencia de esta tesis y otra muy distinta considerar que van Heijenoort logró captar , con su interpretación, las diferencias que Frege pretendió destacar con el uso de los términos 'cálculo' y 'lenguaje caracteristico'. No creo, en efecto, que los textos escritos por Frege para explicar su Begriffsscrift den pie a una interpretación como la ofrecida por van Heijenoort, por sugerente que pueda resultarnos, sino que me parece que, en ellos, los términos 'cálculo' y 'lenguaje caracteristico' desempeñan un papel bien distinto al que van Heijenoort se encarga de destacar. Qué papel es éste y qué distinción es la que Frege quiso subrayar es lo que trataré de analizar en lo que sigue.

\section{La tesis de la prioridad del juicio sobre el concepto.}

El primer elemento que, a juicio de Frege, distingue a su sistema de los de Boole y sus seguidores es el hecho de que, en el suyo, se observa un principio que en los otros no está presente: el de la prioridad de los juicios sobre los conceptos que los componen. Son muchas las ocasiones en las que Frege hace hincapié en esta diferencia a lo largo de estos primeros escritos. Asi, en "Rechnende..." afirma: "En contraposición a Boole, mi punto de partida son los juicios y sus contenidos, y no los conceptos....A la formación de conceptos llego a partir de juicios"13.

$\mathrm{Al}$ asumir semejante principio. Frege rompe con una tradición lógica que se remonta nada menos que a Aristóteles, tradición según la cual los conceptos se forman por abstracción a partir de cosas individuales, siendo previos a los juicios, que no son sino resultado de la agregación de los conceptos componentes previamente dados. A esta tradición pertenecen también Leibniz y Boole

${ }^{13}$ NS. p. 17. 
, y todos sus miembros comparten no solo esta teoria agregacionista del juicio, sino también la idea de que la lógica de clases es mâs básica y fundamental que la de proposiciones, que no es sino una consecuencia de aquella. Que Frege no se engaña en este sentido respecto de Leibniz, se desprende de las siguientes palabras: "Yo no parto de conceptos ni de la idea de que el juicio o pensamiento se forma a partir de ellos, sino que llego a las partes de un pensamiento a través de su análisis. En este sentido mi Begriffsscrift difiere de las creaciones similares de Leibniz y sus sucesores, a pesar de su desafortunado nombre"14.

El primero en mantener la doctrina de que los juicios no se forman a partir de componentes previamente dados, sino que poseen una unidad original y primigenia respecto de los conceptos, a los cuales solo podemos llegar a través del análisis de los juicios, fue un filósofo con el que, a pesar de su equivocada predicción respecto del futuro de la lógica, los fundadores de la lógica moderna están en deuda por alguna que otra importante idea. Hablo naturalmente de Kant, de quien es muy probable que dicha tesis haya pasado a nuestro autor, como muchos otros elementos kantianos de su pensamiento, a través de la obra de Lotze, uno de los filósofos que parece haber ejercido más influencia sobre su pensamiento, a pesar de los recelos y antipatías por él mostradas hacia la formalización de la lógica ${ }^{15}$.

Esta idea de que los juicios son previos a los conceptos guarda. en el caso de Frege, una relación muy estrecha con un principio de carácter metodológico que nos es mucho más familiar: el llamado "principio del contexto", que expresó en los Die Grundlagen der Arithmetik mediante las archiconocidas palabras "Solo en el

14 NS. p. 273. Precisamente el que Leibniz mantiene este punto de vista agregacionista es el argumento fundamental esgrimido por Schröder en favor de su illea de que es el sistema de Boole (y el suyo propio, por tantol el que más se aproxima al ideal leibniziano de lengua universal.

15 Una vez más $M$. Dummett difiere de esta interpretación y mantiene que cualquier intento de vincular a Frege con Lotze es un desafuero histórico ("Frege as a realist". Inquiry. vol. 29 (1976). p. 457). Sin embargo, hay bases más que suficlentes para pensar que ideas como la del logicismo. la de objetividad. o ésta que ahora estamos discutiendo fueron tomadas por Frege de la Logik de Lotze. si bien luego les imprimió su sello propio. 
contexto de una proposición adquiere significado una palabra"16. Este principio lo introduce Frege como segundo principio metodológico para averiguar el sentido de una expresión numérica y advierte que, si se hace caso omiso de él, se contraviene con ello el primer principio, cuya formulación reza "Hay que separar rigurosamente lo psicológico de lo lógico, lo subjetivo de lo objetivo". Se trata, pues, de un principio que, sea cual sea su interpretación correcta -cosa ésta que ha originado muchas disputas ${ }^{17}$ - no puede confundirse con el que venimos analizando. de mucha mayor generalidad y más amplias consecuencias para la construcción del sistema lógico fregeano.

La asunción de esta doctrina de la prioridad del juicio sobre el concepto por parte de Frege es, efectivamente, la causante, por un lado, de la presentación que hará de la lógica en Begriffsscrift. y, por otro, de ciertas particularidades de su simbolismo lógico. En efecto, es la idea de que los juicios son primero que los conceptos, y no al revés, la que le lleva a conferir primacia en su sistema a la lógica de proposiciones sobre la de clases, y a recusar, por tanto. la presentación que Boole hiciera de la lógica,que era una presentación en la que la lógica de clases ocupaba un lugar privilegiado, no siendo la de proposiciones más que una reinterpretación de aquella. Frente a esta concepción booleana del edificio de la lógica, Frege expresa su convicción de que

Basar el 'cálculo de juicios' sobre el 'cálculo de conceptos' (que no es sino un 'cálculo de clases') supone invertir el orden de cosas correcto: pues las clases son algo derivado y solo pueden obtenerse a partir de conceptos (en mi sentido). Pero los conceptos son algo primitivo, de lo que no se puede prescindir en lógica. Sólo podemos determinar una clase especificando las propiedades que un objeto ha de tener para pertenecer a ella. Mas estas propiedades son los atributos (Merkmale) de un

${ }^{16}$ The Foundations of Arithmetic, Oxford, 1959: $2^{\text {a }}$ ed. rev. por Evanston. Ill., Northwestern Univ. Press. 1980. p. X. A partir de ahora me referiré a esta obra como F.

${ }^{17}$ Un análisis bastante completo del principio del contexto, que tanta influencia ejerció en filósofos como Wittgenstein. puede verse en $\mathrm{C}$. Thiel, Sentido y referencia en la lógica de Frege, Madrid. Tecnos, pp. 127 y ss. 
concepto. Definimos un concepto y lo pasamos por alto para ir a la clase. Por esta razón, el cálculo de clases debe basarse en el cálculo de conceptos y este cálculo de conceptos se basa, a su vez, sobre el cálculo de valores de verdad (expresión ésta más adecuada que la de 'cálculo de juicios') ${ }^{18}$.

Consecuente con este modo de pensar, él erigirá todo el sistema, como veremos más detenidamente enseguida, sobre la lógica de proposiciones o -como él dice en su Begriffscrift, en donde todavia no se habla de valores de verdad- de los contenidos judicables (Beurtheilbare Inhalte), relegando la lógica de clases a un papel puramente secundario. A su juicio, esta reorganización de la lógica constituye el único modo de poder hacer ver el vinculo existente entre una y otra parte de la misma y, en consecuencia, de poder ofrecer una presentación unitaria. "En contraposición a Boole, reduzco las proposiciones primarias a secundarias. De este modo creo haber producido de un modo más fácil y apropiado un vinculo orgánico entra ambas partes", afirma en el trabajo tantas veces citado $^{19}$. No podremos comprender, sin embargo, por qué Frege piensa que su sistema tiene esta virtud sin tener presentes los que pasan por ser sus dos más importantes logros en materia de lógica. Me refiero, naturalmente,por una parte, a su propuesta de análisis de las proposiciones en términos de función y argumento, en vez de en términos de sujeto y predicado, y, por otra, a su consideración de las proposiciones generales y consiguiente introducción de la notación correspondiente a los modemos cuantificadores.

Como es sabido, Frege sustituyó la concepción tradicional de la proposición, que consideraba toda proposición como una proposición atributiva , i.e.. como compuesta de sujeto y predicado. por un

18 "Remarks on P. Jourdain 'The development of theories of mathematical logic and the principles of mathematics'. Quarterly Journal of pure and applied Mathematics 43 (1912). p. 252. nota. Frege tamblén critica el modo algebraico de estructurar la lógica a comienzos de sus Grundgesetze y en el ensayo que. en 1895. dedica a la lógica de Schröder, "Kritische Beleuchtung einiger Punkte in E. Schröders 'Vorlesungen über die Algebra der Logik"'. cuya version inglesa puede verse en Collected Papers... ed. cit. pp.210-229.

${ }^{19}$ NS, p. 19. 
interpretación en términos de función y argumento, conceptos que tomó de la matemática. Esta interpretación trata a las proposiciones que atribuyen propiedades a objetos individuales como casos especiales de aquellas en que una función de $n$ - lugares se aplica a $n$ argumentos, justo al revés de cómo hacia la concepción tradicional,para la que todas las proposiciones tenian la misma estructura que las singulares. Estos têrminos permiten un análisis mucho más flexible de la proposición, pero su importancia reside sobre todo en que su introducción llevó a nuestro autor a pergeñar un tratamiento más adecuado de las proposiciones generales ii.e. universales y particulares) y al descubrimiento de la teoria de la cuantificación. El análisis fregeano de las proposiciones generales entraña, como sabe cualquier conocedor de la lógica actual, tres elementos: 1) la distinción entre función y argumento, distinción que le permite considerar los enunciados generales como enunciados acerca de funciones, y no acerca de argumentos; 2) el empleo de variables para expresar la distinción entre lugares determinados e indeterminados en una expresión. y 3) la introducción de una notación especial que permite no solo indicar de manera precisa el alcance de una afirmación de generalidad, sino distinguir entre distintos lugares de argumento en una sentencia con distintas afirmaciones de generalidad. Con estos elementos, además de ser posible un tratamiento más satisfactorio de las proposiciones existenciales que el existente hasta entonces, se puede también dar cuenta de las proposiciones de generalidad múltiple, cosa que no cabia hacer sin ayuda de este recurso que resulta, por tanto, imprescindible para el análisis formal de las proposiciones de la aritmética, el verdadero objetivo perseguido por nuestro autor.

\section{Primacía de la logica proposicional.}

La primera cosa en la que se manifiesta la aceptación por parte de Frege de esta doctrina de la prioridad del juicio, tan profundamente anclada en su pensamiento, es en la organización y presentación que hace de la lógica desde el primer momento, i.e.. desde su primera exposición de la misma en Begriffsscrift. Esta presentación marca para él, como se desprende de su respuesta a la critica de 
Schröder a esta obra ${ }^{20}$, una de las principales diferencias entre su proyecto y el de Boole. En efecto, mientras que en los sistemas construidos por Boole y por sus seguidores, el núcleo fundamental lo constituye la lógica de clases, o lógica de las proposiciones primarias, para Frege la lógica primaria y fundamental, sobre la que se asienta todo lo demás, es, por el contrario, la lógica proposicional o. como él la denomina en esta primera etapa de su pensamiento, de los contenidos judicables no analizados.

Frege parte del supuesto de que estos contenidos judicables pueden ser de dos clases, afirmados y negados, o. como después de su introducción de la distinción entre sentido y referencia preferirá decir, verdaderos y falsos ${ }^{21}$. Este cambio de denominación no tiene, sin embargo, demasiada importancia, dado que Frege usa los términos 'afirmado' y 'negado' en Begriffsscrift con el mismo sentido que posteriormente conferirá a los de 'verdadero' y 'falso' en el sistema lógico presentado en el primer volumen de sus Grundgesetze. La razón es que los dos principios que van a gobernar su lógica siempre, con independencia de esta distinta forma de hablar, son el de que los zontenidos judicables o bien son afirmados o bien son negados, pero no ambas cosas a la vez, y el de que el valor de verdad de un contenido complejo está determinado por el valor de verdad de los componentes del mismo. También el sistema de Boole parte de la asunción de estos dos supuestos de bivalencia y funcionalidad veritativa, pero aqui se acaban todas las semejanzas, pues el hecho de que uno acepte el principio de prioridad mientras que el otro no, junto con el distinto propósito que, como luego veremos, subyace a sus respectivos proyectos, acarreará aún alguna otra diferencia más entre los sistemas de ambos.

Una que enseguida salta a la vista es que en uno y otro sistema se eligen como fundamentales distintas operaciones. En efecto,

\footnotetext{
20 "On the aim of the 'Conceptual Notation"'. CP. pp 90-101.

21 La distinción entre sentido y referencia, realizada en "Über sinn und bedeutung", le llevará a dividir la noción de 'contenido judicable' en pensamiento. que no es sino el sentido de una sentencia asertórica y valor de verdad, que es la referencia de la misma. Este caubio de denominación lo explica. entre otros lugares. además del antedicho, en "On concept and object' CP, p. 187 y en Grundgesetze (versión inglesa incompleta. The Basic Laws of Arithmetic, Berkeley y Los Angeles. Univ. of California Press. 1964, p. 38)
} 
mientras que en el sistema de Boole las operaciones seleccionadas son las de negación, conjunción y disyunción, que son las más idóneas para la construcción de un cálculo, que es lo que èl iba buscando, en el de Frege la operación que goza de un estatuto privilegiado es el condicional, por considerarse más idónea para la expresión de relaciones inferenciales, que es en lo que Frege está verdaderamente interesado.

No fue Frege, sin embargo, el primer autor de la lógica moderna que hizo de la implicación la relación lógica fundamental ni tampoco el primero que confirió primacia a la lógica proposicional sobre la de clases, sino que este mérito le corresponde en realidad a uno de los lógicos más injustamente olvidados de toda la historia de la evolución de la lógica. Me refiero al escocés Hugh MacColl, el cual, en fecha tan temprano como 1877 inició la publicación de una serie de trabajos en los que presenta un sistema al que denomina de 'enunciados equivalentes', que en realidad no es otra cosa que una lógica de proposiciones no analizadas ${ }^{22}$. En este sistema, la operación lógica fundamental es también la relación de implicación, que se representa mediante $a: b$ (en donde $a$ y $b$ son variables proposicionales), lo que ocurre es que esta noción no se interpreta de un modo veritativo-funcional, sino en un sentido que recuerda al que poco después otorgará Lewis a la relación de implicación estricta, en torno a la cual surgirán las primeras lógicas modales $^{23}$.

No será asi como nuestro autor interprete el condicional, sino que, para él, la expresión "Si B entonces A" tiene pura y simplemente el sentido "no (B y no-A)" . En efecto, teniendo en cuenta que, dados dos contenidos judicables,son cuatro las combinaciones posibles que cabe distinguir, a saber.

${ }^{22}$ En el año 1877 MacColl inicia la publicación. en los Proceedings of the London Mathematical Society. de una serie de slete articulos. todos los cuales llevan por titulo "The calculus of equivalent statements". serle que se completa con otra de ocho, publicados en la revista Múrd entre los años 1880-1906. bajo el título genérico de "Symbolic Reasoning".

${ }^{23}$ Vide, por ejemplo. Mind V (1880). p. 54, MInd VI (1897), p. 507. Mind XII (1903). p. 262 y Mind XVII (1908). p. 152. 
A es afirmado y $B$ es afirmado.

A es afirmado y $B$ es negado.

A es negado y $B$ es afirmado, $y$

A es negado y $B$ es negado,

mediante la relación condicional, Frege expresa el juicio "la tercera de estas posibilidades no se realiza, sino una de las otras tres", siendo por tanto su condicional del todo similar a la implicación filónica de la antigüedad.

Con ayuda de esta operación y de la negación logra Frege la hazaña de establecer, con una precisión formal dificilmente superable, la primera formulación axiomática de esta lógica, formulación que, además, como se probará más tarde, resulta ser completa, i.e., todas las proposiciones válidas de la lógica proposicional bivalente son derivables de los seis axiomas por él seleccionados.

Entre tanto, la suerte corrida por la lógica proposicional entre los miembros de la tradición algebraica irá sufriendo variaciones. Por una parte, los primeros seguidores de Boole, S.Jevons y J. Venn, se mantienen fieles a las directrices booleanas de construir un cálculo cuya interpretación principal es una lógica de clases. de forma que la lógica proposicional sigue siendo para ellos secundaria por respecto de aquella. Que esta lógica no es para ellos sino una mera reinterpretación de la de clases, es algo que se desprende no solo de los sistemas por ellos construidos, que no introducen sino ligeras mejoras en el álgebra de Boole, sino también de lo poco favorable de los comentarios que a uno y otro les merece la obra lógica de MacColl, en la que se prima a la proposición sobre la clase. Pero, por otra parte, están los casos del lógico americano C. Peirce y de Schröder, cuya obra está ya bastante lejos de ser una mera reproducción de este viejo esquema. El primero de ellos empieza adhiriéndose al cálculo ecuacional caracteristico de los sistemas de los anteriores, pero ya en 1870 decide sustituir la relación de identidad por la de inclusión de clases, que a su vez abandonará en beneficio del condicional en la presentación de un cálculo que él denomina "lógica no-relativa", pero que no es otra cosa que una lógica proposicional del corte de las de tipo no- 
algebraico ${ }^{24}$. La tarea de obtener, desde una perspectiva algebraica, la lógica proposicional a partir de la de clases, utilizando la idea de que las proposiciones se caracterizan por ser verdaderas o falsas, quedará para Schröder, el cual la lleva a cabo en el segundo de los tres volúmenes que componen sus Vorlesungen über die Algebra der Logik ${ }^{25}$.

Pero las operaciones que se eligen como básicas en uno y otro sistema no son las únicas diferencias apreciables que se siguen de los distintos presupuestos y proyectos de Boole y de Frege. Los sistemas lógicos presentados por uno y otro difieren aún en otra cosa, a saber, el sistema notacional empleado. Frente al carácter secuencial y lineal de la escritura booleana, Frege adopta, en cambio, un sistema bidimensional, i. e.. un sistema en el que las diversas proposiciones o formas proposicionales unidas por conectivas se escriben cada una en una fila distinta, con las conectivas formando un entramado de lineas a la izquierda de la secuencia. Este sistema fue tachado de "engorroso e inadecuado" por Venn y considerado por Schröder como un "monstruoso gasto de espacio"26, mas Frege lo defiende de tales intentos de desacreditación alegando, de una parte. que permite captar mejor las relaciones exhibidas y, en este sentido, es más claro que el lineal, y, de otra que, dado el objetivo que él persigue de reducir la matemática a lógica, resulta si no insoslayable, al menos mucho más conveniente que el de Boole, pues en éste se confiere a los símbolos matemáticos un cometido lógico, cosa que êl no puede hacer porque los necesita para la expresión de relaciones

24 Peirce introduce la relación de inclusion en "Description of a notation for the logic of relatives resulting of a amplification of Boole's calculus of logic". en Writings of Charles S. Peirce: a Chronological edition. vol. 2. (ed. E. Moore, Bloomington, Indiana Univ. Press, 1984. p. 360, nota 1. Tras un periodo de confusión respecto a la interpretación de esta relación. en su "On the algebra of logic". de 1885. (versión española en C.Peirce: Escritos lógicos , ed. P. Castrillo. Madrid. Alianza Editorial. 1988), logra ya la presentación de un sistema muy similar al de Begriffsscrift. i.e.. que ya nada tiene que ver con los algebraicos.

${ }^{25}$ Este volumen se publicó en 1891 , después de un primero dedicado a la lógica de clases. El tercero y último de la serie. de 1895. está dedicado a la lógica de relaciones.

${ }^{26} C N$, p. 234 y p. 219 , respectivamente. 
matemáticas ${ }^{27}$. Esto quiere decir que el desacuerdo entre ellos acerca de la notación no se reduce a un puro desacuerdo en materia tipográfica, sino que refleja una concepción distinta de las relaciones entre matemática y lógica $y$, en definitiva, un distinto proyecto subyacente a las construcciones de ambos.

\section{El proyecto logicista}

El propósito que Frege persiguió a lo largo de toda su obra es el de construir un lenguaje en el que poder expresar las proposiciones de la matemática con más precisión que en el lenguaje ordinario y en el que poder determinar las bases sobre las que se sustenta la verdad matemática. Este proyecto, que no es otro que el proyecto logicista, está totalmente ausente de la obra de Boole y sus seguidores, que no pretendieron reducir la matemática a lógica, sino simplemente construir la lógica como un cálculo más de la matemática. No tiene nada de extraño, pues, dada la importancia que para él tiene este proyecto, que Frege haya visto aqui otra de las grandes divergencias entre su sistema y el de Boole. Según su interpretación, el objetivo de Boole es construir una lógica pura, un cálculo abstracto, en tanto que el que él persigue es el de construir un sistema apto para la expresión de la matemática; por eso el suyo es una lingua characterica, en tanto que el de aquel no es más que un calculus ${ }^{28}$.

Lo que Frege pretendia, en efecto, era mostrar que las proposiciones de la aritmética no son ni simbolos vacios, ni generalizaciones inductivas ni leyes psicológicas del pensamiento, sino verdades que no solo son a priori sino que además son analíticas. Este programa, consistente. pues. en reducir la aritmética a la lógica o en mostrar que aquella no es sino una parte propia de ésta. constituye el hilo conductor de toda la obra de Frege hasta que tiene lugar el descubrimiento, por parte de Russell, de que del axioma V de sus Grundgesetze podia derivarse una contradicción. descubrimiento que supuso el fracaso del programa logicista y que

\footnotetext{
27 "On the aim...", CN. pp. 90-101, y "Remarks on P. Jourdain...". op. cit., p. 253.

28 "Booles rechnende...". NS. p. 13 y "On the aim ...", CN, p 97.
} 
acabó con las esperanzas de Frege de poder realizarlo tal y como él lo habia ideado. Conviene precisar que Frege daba importancia a su reducción sólo en la medida en que confiaba en que era una reducción a la lógica: tan pronto como perdió la esperanza de hallar un medio relativamente simple de evitar la contradicción, se entregó a la idea de que habia sido un error tratar la teoria de clases como una parte de la lógica, y la reducción dejó de interesarle.

De las reflexiones filosóficas contenidas en sus Fundamentos de la aritmética (Grundlagen), obra que compuso antes de emprender la tarea de explicar cómo se pueden definir los conceptos de esta ciencia en términos exclusivamente lógicos, parece poder inferirse que lo que indujo a Frege a tratar de desarrollar este proyecto logicista fue la idea de poder poner de manifiesto la verdad del apriorismo kantiano, asi como de sus tesis acerca de la naturaleza y limites del entendimiento humano. No es que Frege pensara que Kant estaba en lo cierto acerca de la naturaleza de los juicios de la aritmética, pues los habia caracterizado como sintéticos, siendo asi que êl los consideraba analiticos, pero creia que este error de Kant no era nada comparado con la magnitud de lo que habia aportado estableciendo su carácter apriórico. En concreto, escribe

Por mi parte, no quiero hacerme acreedor del reproche de molestar con minucias a un genio a quien todos debemos mirar con agradecido respeto. Me veo, pues, en la obligación de llamar la atención sobre el alcance de mis coincidencias con él, que supera en mucho al de mis discrepancias. Para circunscribirme a lo que aqui estamos discutiendo, pienso que Kant prestó un gran servicio al establecer la distinción entre juicios analíticos y sintéticos. Al tomar las verdades de la geometria por sintéticas y a priori puso de manifiesto su auténtica naturaleza. Y ello merece repetirse aún, dado que en nuestros dias no siempre se le reconoce. Si se equivocó en lo que respecta a la aritmética, ello no resta valor a su obra. Su propósito era hacer ver la posibilidad de juicios sintéticos a priori en el dominio de la matemática; que éstos se den en la geometria o también en la aritmética, tiene ya menos importancia ${ }^{29}$.

${ }^{29}$ F. p. 101 
El proyecto global fregeano estaba, pues, motivado por intereses epistemológicos y, en cierto modo, también kantianos. Frege estaba interesado en interpretar la matemática dentro de un marco epistemológico que tomó de Kant ${ }^{30}$. En sus Fundamentos de la aritmética. Frege operó con la idea de que en realidad no hay más que un pequeño número de posibilidades de hallar la justificación de los enunciados aritméticos, y adoptó la estrategia de la eliminación de alternativas en favor de la tesis logicista. La distinción analitico/sintético, en combinación con la distinción a priori/a posteriori, le permite categorizar las proposiciones respecto al fundamento último que cabe invocar para su justificación. La división básica para él es la que media entre procedimientos justificatorios a priori y a posteriori. Dentro de la categoria de los segundos, cabe distinguir aún dos clases: la intuición interna y el pensamiento: i.e., se puede pensar que la aritmética se basa en alguna intuición a priori, o que es derivable de la lógica. Esta última alternativa es el logicismo y Frege arguye en favor de ella siguiendo el procedimiento de ir descartando las otras, i.e., la de que las proposiciones aritméticas tengan justificación empirica y la de que haya alguna intuición a priori disponible en el caso de la aritmética. De hecho Frege arguye también, aunque menos explícitamente, contra otra posibilidad: la de considerar las proposiciones matemáticas como asignificativas, toda vez que alli se considera la matemática como un cálculo con un contenido concreto.

La posibilidad que a Frege le merece más respeto, aparte del logicismo, es la que considera que la aritmética se basa en alguna intuición a priori. i.e., la representada por Kant. De hecho, compartirá hasta el final de sus dias con este filósofo una postura

${ }^{30}$ La tesis de que la motivación subyacente al proyecto de Frege es kantiana ha sido mantenida en los ültimos tiempos, entre otros, por H. Sluga, Gottlob Frege, Routledge and Kegan Paul, London. 1980:T. Burge. "Sinning against Frege". Philosophical Review88 (1979), 398-432: P. Kitcher, "Frege's Epistemology", ibid. pp. 235-262 y G. Currie. Frege:An introduction to his philosophy, Totowa.N.J Barnes and Noble, 1982. 
similar a la suya a propósito de la geometria ${ }^{31}$. En lo que respecta a la aritmética, él piensa, sin embargo, que, si bien muchas demostraciones parecen contener apelaciones a la intuición, tales apelaciones son engañosas, ya que muchas veces simplemente indican que la transición al paso siguiente es obvia. Parte del proyecto fregeano consiste en establecer si la apelación a la intuición es simplemente una indicación de que la transición a la linea siguiente requiere una serie de pasos no explicitados por mor de la claridad o si mas bien depende de alguna presuposición no establecida, como ocurre, por ejemplo, según nos dice, en algunas pruebas de los Elementos de Euclides ${ }^{32}$. Es precisamente esta parte del proyecto la que le llevó a desarrollar el lenguaje de $B e$ griffsscrift, toda vez que el lenguaje natural es insuficiente para la realización de esta tarea, y es en este punto en donde . en consecuencia, más se parta su proyecto del construido por los booleanos. más preocupados por hacer cuadrar una interpretación lógica en unos esquemas excesivamente apegados a sus modelos matemáticos.

Durante las primeras tres décadas del presente siglo, la filosofia de la matemática ha estado dominada por tres programas de investigación que se corresponden con tres de las cuatro posibilidades contempladas por Frege. El más próximo al enfoque de éste es el emprendido por Russell y Whitehead en Principia Mathematica, si bien alguno de los principios que adoptaron como punto de partida no es incontrovertiblemente lógico. El segundo, el formalismo de Hilbert, constituye un desarrollo de la idea de que la matemática es un juego formal. Y, en cuanto al tercero, el intuicionismo, desarrollado por Brouwer, mantiene la tesis de que el fundamento del conocimiento matemático estriba en la intuición a priori. El único programa que está ausente del panorama de los primeros años del siglo XX es el programa empirista, precisamente aquel que más tinta hiciera gastar a nuestro autor, empeñado sobre todo en oponerse al naturalismo de su tiempo en todas sus

\footnotetext{
${ }^{31}$ Al final de su vida, después del fracaso del programa logicista, llegó incluso a considerar artificial y equivocada la separactón que habia establecido entre la aritmética y la geometria y a buscar un fundamento en ésta última. Vide. NS. p.297.

32 "On the scientific justification..". CN. p. 85.
} 
modalidades, y en establecer la verdad de la afirmación de que no todo conocimiento es empirico. Determinar en qué medida sus preocupaciones fueron las mismas que las de los neokantianos de su época y hasta qué punto forma parte, por tanto, de la tradición idealista alemana o más bien el uso por parte de Frege de la terminologia y esquemas kantianos se debe únicamente a un intento de hacerse entender por los filósofos profesionales de su tiempo es una tarea que urge llevar a cabo $^{33}$. pero que transciende el objetivo de este trabajo.

Resumiendo lo dicho: la doctrina de la prioridad del juicio sobre el concepto, junto con la idea de la reducción de la aritmética a la lógica, confirieron al proyecto fregeano de construcción de un lenguaje caracteristico unas peculiaridades que estaba lejos de poseer el de Boole y sus seguidores. Frege fue claramente consciente de las diferencias que separaban a uno de otro proyecto y de las novedades que encerraba su sistema, e hizo claramente explicitas unas y otras en estos escritos explicativos que siguieron a la publicación de su Begriffsscrift. El destinatario directo de los mismos, Schröder, siguió, sin embargo, sin entender, como lo demuestra el hecho de que quince años después, en un trabajo en que hacia una valoración del estado de la lógica en Italia, escribiera todavia $^{34}$

Dejando estas investigaciones concretas de origen inglés y pasando por alto varios intentos aislados (como, por ejemplo, el del Sr. Frege, quien haciendo caso omiso de todo lo logrado en la misma dirección por otros, dedicó grandes esfuerzos a realizar lo que ya habia sido hecho de un modo mucho mejor, $y$ fue por consiguiente, suplantado desde el principio, alumbrando de este modo un niño ya nacido), con la excepción

33 Las dos posturas extremas estarian representadas. por el momento, de un lado. por Sluga, quien en el capitulo 2 de su Gottlob Frege. antes citado, nos presenta a Frege como un miembro perteneciente a la tradición idealista y como alguien integrado en la corriente de pensamiento de su época. $y$, de otro, por Dummett. para quien parece como si la lógica fregeana hubiese surgido de un cerebro no contaminado por influencias extemas (vide. Frege. The Logic of Language, op. cit., p. XXVII)

34 "On Pasigraphy. Its present state and the pasigraphic movemente in Italy".. The Monist 9 (1898). pp. 44-62. 
del trabajo fundamental del Sr. Peirce en los Estados Unidos y de sus precursores alemanes e ingleses, entre los que cabe destacar a Boole y De Morgan, los objettvos de la Pasigrafia solo han hallado hasta ahora asiduos promotores en Italia ${ }^{35}$.

Por lo demás, la ceguera y falta de generosidad a la hora de valorar las aportaciones de otros no es privativa de Schroder, sino que suele ser moneda bastante común. El propio Frege, cuya reacción ante el anuncio de Russell de que su sistema encerraba una contradicción pasa por ser un modelo de honestidad intelectual, dió también. sin embargo, grandes muestras de falta de generosidad en su apreciación de proyectos como los de Hilbert o Cantor que tanto juego habrian de dar después.

35 Ibid. pp. 60-61. 